\title{
Detection of Inducible Clindamycin Resistance with Erythromycin in Clinical Isolates and Its Prevalence among Methicillin Resistant Staphylococcus aureus
}

\author{
S. Dhivya Lakshmi* and Chitralekha Saikumar
}

Sree Balaji Medical College and Hospital, New colony, Chennai- 600044, India

*Corresponding author

\begin{tabular}{|c|c|}
\hline & $\Gamma$ \\
\hline & \multirow{5}{*}{$\begin{array}{l}\text { Clindamycin is a substitute choice for mild to moderate Staphylococcal infections } \\
\text { especially in penicillin-allergic patients. Staphylococcus aureus is one of the most } \\
\text { common pathogen with ability to cause wide range of infections in humans. Clindamycin } \\
\text { has been used to treat serious infections for more than } 30 \text { years in children. It remains } \\
\text { effective for many infections caused by community-acquired and hospital acquired } \\
\text { methicillin-resistant Staphylococcus aureus (CA-MRSA/ HA- MRSA). The clinical } \\
\text { manifestations of a positive D-test begin with a perception of cross resistance among three } \\
\text { antibiotic families that share a common binding site- Macrolides (e.g., Erythromycin) } \\
\text { Lincosamide (e.g.; Clindamycin), and group B Streptogrammins. A positive D-test } \\
\text { indicates the presence of MLSBi genotype, though, sub inhibitory concentration of } \\
\text { Erythromycin is a common inducer of Inducible Clindamycin resistance (ICR). When } \\
\text { Erythromycin diffuses on a plate streaked with Staphylococcus aureus, it induces, } \\
\text { resistance to Clindamycin and results in flattening of the Clindamycin zone of inhibition } \\
\text { just next to the Erythromycin disk, making a D shape, so this method is called D- test. } \\
\text { Susceptibility testing was performed according to CLSI (Clinical and laboratory standard } \\
\text { Institute) on isolates of Staphylococcus aureus by using the method of disk diffusion. } \\
\text { Almost one third of erythromycin resistant strains have expressed inducible clindamycin } \\
\text { resistance. MRSA isolates has higher inducible clindamycin resistance than MSSA. } \\
\text { Henceforth D test becomes a vital part in routine laboratory procedures for the detection of } \\
\text { inducible resistance than leading to therapeutic failure. }\end{array}$} \\
\hline & \\
\hline Artic & \\
\hline & \\
\hline & \\
\hline
\end{tabular}

\section{Introduction}

Staphylococcus aureus (S. aureus) causes varied range of infections in humans counting nosocomial and community-acquired infections in every part of the world. The increase incidence of MRSA is an emergent problem (Yilmaz et al., 2007). Even though healthy children have a small risk for serious infections, the children might be common carriers of this organism. Numerous clinical infections may develop as well as spread from nasal carrier individuals.

Very few alternating antibiotics options were left behind for the treatment of methicillin resistance Staphylococcal infections. One of such effective alternative is macrolide- 
lincosamide - streptogramin B $\left(\mathrm{MLS}_{\mathrm{B}}\right)$ family of antibiotics. Of these, macrolide group (Erythromycin) and lincosamide class (Clindamycin), that inhibit protein synthesis by binding to the $50 \mathrm{~S}$ ribosomal subunits of bacteria as their mechanism of action against the organisms.

With excellent pharmacokinetic properties, Clindamycin has taken over as the most ideal antibiotic. (Delialioglu et al., 2005) However, extensive use of $\mathrm{MLS}_{\mathrm{B}}$ antibiotics has led the strains to acquire resistance to these antibiotics (Deotale et al., 2010; Ajantha et al., 2008).

Staphylococcus aureus offers resistance to these two distinct classes of antimicrobial agents through methylation of their ribosomal target site. This Target site modification mechanism of resistance is mediated by erm genes. The resistance can be expressed either constitutively (constitutive MLS B phenotype) or inducibly (inducible MLS B phenotype) (Steward et al., 2005).

Strains with inducible clindamycin resistance, when not placed adjacent to each other become difficult to detect in the routine laboratory as they appear erythromycin resistant and clindamycin sensitive. In such cases, clindamycin may select constitutive erm mutants leading to clinical therapeutic failure. Another mechanism of resistance, efflux of antibiotic which is mediated through $m s r A$ genes, Staphylococcal isolates appear erythromycin resistant and clindamycin sensitive both in vivo and in vitro and the strain does not typically become clindamycin resistant during therapy

This study is conducted to find the prevalence of Erythromycin resistant Staphylococcus aureus strains and its antibiotic susceptibility pattern with clindamycin by doing a feasible $\mathrm{D}$ test and also to elicit the relationship between MRSA and Erythromycin resistance.

\section{Materials and Methods}

The study was conducted from April 2017 to July 2017. A total of $180 \mathrm{~S}$. aureus were isolated from various clinical specimens like pus, wound swab, aspirates, blood, and sterile fluids and tested. The isolates were first identified as $S$. aureus by conventional standard biochemical techniques (Milles and Amyes, 1999) and then subjected to susceptibility testing by modified Kirby Bauer's disc diffusion method on Mueller Hinton agar plates using erythromycin (15 $\mu \mathrm{g})$, clindamycin $(2 \mu \mathrm{g})$, vancomycin $(30 \mu \mathrm{g})$, and cefoxitin $(30 \mu \mathrm{g})$ as per CLSI guidelines (Clinical and Laboratory Standards Institute, 2017). An inhibition zone of $19 \mathrm{~mm}$ or less around cefoxitin disc indicates MRSA.

' $\mathrm{D}$ test' to test Inducible resistance to clindamycin was done as per CLSI guidelines (Clinical and Laboratory Standards Institute, 2017). On previously inoculated on MuellerHinton agar plate with $1 \mathrm{McFarland}$ standard bacterial suspensions, Erythromycin $(15 \mu \mathrm{g})$ disc was placed at a distance of $15 \mathrm{~mm}$ (edge to edge) from clindamycin $(2 \mu \mathrm{g})$.

Following overnight incubation at $37^{\circ} \mathrm{C}$, three different types of erythromycin resistance pattern was observed. They are as follows

MS phenotype - Isolates showing resistance to erythromycin (zone size $\leq 13 \mathrm{~mm}$ ) while susceptible to clindamycin (zone size $\geq 21$ $\mathrm{mm}$ ) with regular, circular shape zone of inhibition around clindamycin belongs to this phenotype.

Inducible $\mathrm{MLS}_{\mathrm{B}}\left(\mathrm{iMLS}_{\mathrm{B}}\right)$ phenotype - Isolates exhibiting resistance to erythromycin (zone size $\leq 13 \mathrm{~mm}$ ) while being susceptible to clindamycin (zone size $\geq 21 \mathrm{~mm}$ ) and with flattening towards erythromycin disc giving D-shaped zone of inhibition around clindamycin belongs to this phenotype. 
Constitutive $\mathrm{MLS}_{\mathrm{B}}$ phenotype - this phenotype was labeled for those Staphylococcal isolates, which showed resistance to both erythromycin (zone size $\leq 13 \mathrm{~mm}$ ) and clindamycin (zone size $\leq 14 \mathrm{~mm}$ ) with circular shape of zone of inhibition if any around clindamycin.

\section{Results and Discussion}

Total number of strains included in the study is 180 . Out of this 180 isolates erythromycin resistance and clindamycin resistance were observed in $69(38.3 \%)$ and 28 (15.5\%) respectively. Among erythromycin resistant isolates, clindamycin was resistant in 9 (13\%) isolates which accounts for the Constitutive $\mathrm{MLS}_{\mathrm{B}}$ Phenotype. Total clindamycin susceptibility was observed in 152 isolates. Among clindamycin susceptible strains, erythromycin resistance was seen in 60 isolates.

D test positive $-22(31.8 \%)$

(Inducible $\mathrm{MLS}_{\mathrm{B}}$ Phenotype)

D test negative $-38(55 \%)$

(MS Phenotype)

Wide range of infections are caused by Staphylococcus aureus in humans. The occurrence of invasive infections has been rising with emergence of Hospital Acquired (HA) and community acquired (CA) Methicillin Resistant Staphylococcus aureus. (MRSA). Clindamycin claims to be the one of the effective alternative antimicrobial agent for treating Methicillin resistant staphylococci infections mainly in soft tissue infections. Though the claim to be, it has to be investigated prior for its hidden inducible resistance along with erythromycin to prevent clinical failure of the treatment. On the alternative, negative inducible clindamycin resistance settles clindamycin susceptibility and provides a very good therapeutic option as it has a good oral bioavailability (Deotale $e t$ al., 2010). In routine antimicrobial susceptibility test for all $S$. aureus D-test becomes a domineering part (Gupta et al., 2009) because by using standard susceptibility test methods the $\mathrm{iMLS}_{\mathrm{B}}$ resistance mechanism is not predictable also its prevalence differs according to geographic location.

In our study, the erythromycin resistant were found in $69(38.3 \%)$ isolates. Similar studies by (Prabhu Kavitha, 2017) and (Deotale et al., 2010) reported erythromycin resistance to be $28.42 \%$ and $32.4 \%$ respectively, which actually quotes on the higher range.

Assessment of erythromycin resistance among MRSA and MSSA

\begin{tabular}{|l|l|l|l|l|}
\hline S. No. & Erythromycin Pattern & MRSA $(\mathbf{n = 5 6})$ & MSSA $(\mathbf{n = 1 2 4})$ & TOTAL \\
\hline 1. & Constitutive MLS $_{\mathrm{B}}$ & $6(10.7 \%)$ & $3(2.4 \%)$ & 9 \\
\hline 2. & iMLS $(\mathrm{D}+)$ & $16(28.5 \%)$ & $6(4.8 \%)$ & 22 \\
\hline 3. & MS phenotype & $27(48.2 \%)$ & $11(8.8 \%)$ & 38 \\
\hline
\end{tabular}

Among erythromycin resistance isolates, around $32 \%$ were tested positive for inducible clindamycin resistance with a positive $\mathrm{D}$ test. $D$ test negative were seen in 47 strains of which constitutive resistance and MS phenotype accounted for 38 (55\%) and 9 (13\%) respectively. Almost one third of erythromycin resistant strains have projected inducible clindamycin resistance and if this D test is not performed routinely, it would eventually lead to therapeutic failure. Similar findings were obtained in other studies too by (Ciraj et al., 2009).

The assessment of Erythromycin susceptibility pattern among MRSA and 
MSSA also showed that MRSA isolates has upper range of all three types of erythromycin resistance. Inducible $\mathrm{MLS}_{\mathrm{B}}$ and $\mathrm{MS}$ phenotype in MRSA were $28.5 \%$ and $48.2 \%$, respectively which are higher when compared to MSSA (4.8\% and $8.8 \%$ ). Similar study by (Yilmaz et al., 2007) found inducible MLS $_{\mathrm{B}}$ resistance of $24.4 \%$ in MRSA and $14.8 \%$ in MSSA; (Rahabar and Hajia, 2007) reported $22.6 \%$ in MRSA and $4 \%$ in MSSA. On the conflicting side, Schreckenberger et al., (2004) and Levin et al., (2005) showed higher percentage of inducible resistance in MSSA as compared to MRSA.

In our study Constitutive $\mathrm{MLS}_{\mathrm{B}}$ resistance was seen in $10.7 \%$ of MRSA isolates and $2.4 \%$ in MSSA, which is similar to other studies by Deotale et al., (2010) (7.3\% of MRSA isolates). Also contrary to this result, the only study that was reported by Angel et al., from India (Angel et al., 2008) which did not find it in any of the strains

Antibiotic options for the treatment of Methicillin resistance Staphylococcal infections are very limited. Of which Vancomycin would be reserved for life threatening infections. Hence, clindamycin should be considered for the controlling of serious soft tissue infections with MRSA that are truly sensitive to clindamycin. Though, the effectiveness of the drug is restricted by the expression of inducible clindamycin resistance.

So, clinical microbiology laboratories should perform the simple, supporting and reliable D test to report and to differentiate between inducible and constitutive clindamycin resistance routinely in all Staphylococcal isolates that are isolated from all samples received in the laboratories. Likewise D test enable us in guiding the clinicians regarding judicious use of clindamycin in skin and soft tissue infections.

\section{References}

Yilmaz G, Aydin K, Iskender S, Caylan R, Koksal I. Detection and prevalence of inducible clindamycin resistance in staphylococci. J Med Microbiol. 2007; 56:342-5.

Delialioglu N, Aslan G, Ozturk C, Baki V, Sen S, Emekdas G. Inducible clindamycin resistance in staphylococci isolated from clinical samples. Jpn J Infect Dis. 2005; 58:104-6.

Deotale V, Mendiratta DK, Raut U, Narang P. Inducible clindamycin resistance in Staphylococcus aureus isolated from clinical samples. Indian $\mathbf{J}$ Med Microbiol. 2010; 28:124-6.

Ajantha GS, Kulkarni RD, Shetty J, Shubhada C, Jain P. Phenotypic detection of inducible clindamycin resistance among Staphylococcus aureus isolates by using the lower limit of recommended interdisk distance. Indian J Pathol Microbiol. 2008; 5 1:376-8.

Steward CD, Raney PM, Morrell AK, Williams PP, McDougal LK, Jevitt L, et al., Testing for induction of clindamycin resistance in erythromycin resistant isolates of Staphylococcus aureus. J Clin Microbiol 2005; 43:171621.

Milles, R.S., and S.G.B. Amyes. Laboratory control of antimicrobial therapy in Mackie and McCartney, Practical Medical microbiology14th ed. Edited by JG Collee, AG Fraser, P Marmion, A Simmons. 1999. Churchill livingstone. 1996. p151-157.

Clinical and Laboratory Standards Institute. Performance standards for antimicrobial testing; twenty third informational supplement. CLSI document M100S27, Clinical and Laboratory Standards Institute, Wayne, PA. 2017.

Gupta V, Datta P, Rani H, Chander J. Inducible clindamycin resistance in 
Staphylococcus aureus: A study from North India. J Postgrad Med. 2009; 55: 176-9.

Prabhu Kavitha, Sunil Rao, and Venkatakrishna Rao. Inducible Clindamycin Resistance in Staphylococcus aureus Isolated from Clinical Samples. Journal of Laboratory Physicians 3.1 (2011): 2527. PMC. Web. 23 Sept. 2017.

Ciraj AM, Vinod P, Sreejith G, Rajani K. Inducible clindamycin resistance among clinical isolates of Staphylococci. Indian J Pathol Microbiol. 2009; 52:49-51.

Rahabar M, and Hajia M. Inducible clindamycin resistance in Staphylococcus aureus: A cross sectional report. Pak J BiolSci 2007; 10:189-92.

Schreckenberger PC, Ilendo E, Ristow KL.
Incidence of constitutive and inducible clindamycin resistance in Staphylococcus aureus and coagulasenegative staphylococci in a community and a tertiary care hospital. J. Clin. Microbiol., 2004; 42:2777-9.

Levin TP, Suh B, Axelrod P, Truant AL, Fekete T. Potential clindamycin Resistance in clindamycin-susceptible, erythromycin-resistant Staphylococcus aureus: Report of a clinical failure. Antimicrob Agents Chemother 2005; 49:1222-4.

Angel MR, Balaji V, Prakash JA, Brahmandathan KN, Mathews MS. Prevalence of inducible clindamycin resistance in gram positive organisms in a tertiary care centre. Indian $\mathrm{J}$ Med Micobiol 2008; 26:262-4

\section{How to cite this article:}

Dhivya Lakshmi, S. and Chitralekha Saikumar. 2018. Detection of Inducible Clindamycin Resistance with Erythromycin in Clinical Isolates and Its Prevalence among Methicillin Resistant Staphylococcus aureus. Int.J.Curr.Microbiol.App.Sci. 7(01): 2405-2409. doi: https://doi.org/10.20546/ijcmas.2018.701.289 